
\title{
$\begin{array}{ll}\text { Research Square } & \begin{array}{l}\text { Preprints are preliminary reports that have not undergone peer review. } \\ \text { They should not be considered conclusive, used to inform clinical practice, } \\ \text { or referenced by the media as validated information. }\end{array}\end{array}$
}

\section{Large-scale placenta DNA methylation mega-analysis reveals fetal sex- specific differentially methylated CpG sites and regions}

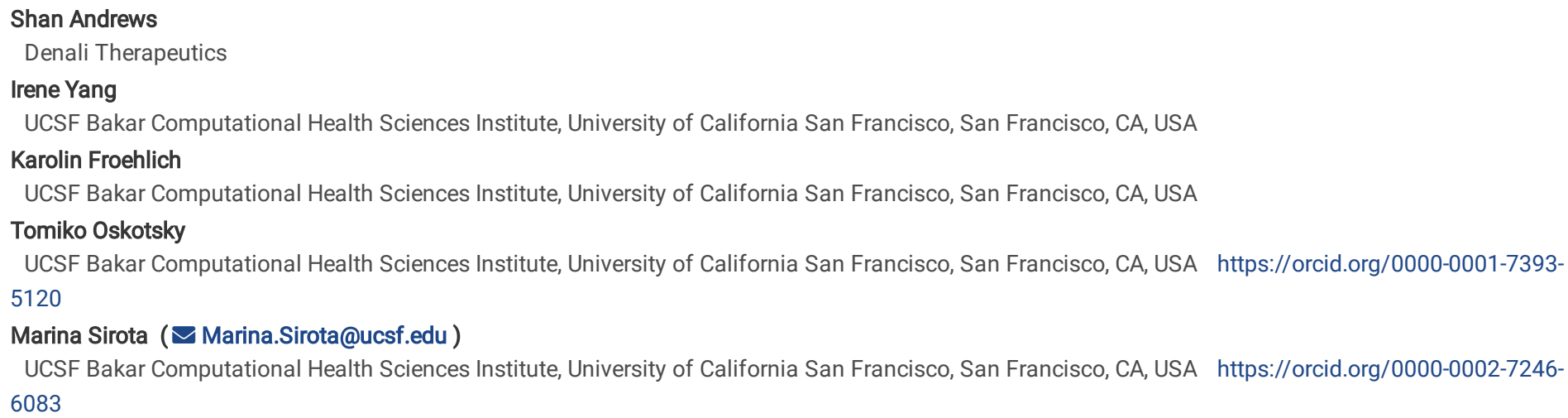




\section{Abstract}

Although male-female differences in placental structure and function have been observed, little is understood about their molecular underpinnings. Here, we present a mega-analysis of 14 publicly available placenta DNA methylation (DNAm) microarray datasets to identify individual CpGs and regions associated with fetal sex. In the discovery dataset of placentas from full term pregnancies ( $N=532$ samples), 5,212 CpGs met genome-wide significance ( $p<1 \mathrm{E}-8)$ and were enriched in pathways such as keratinization (FDR p-value $=7.37 \mathrm{E}-14)$, chemokine activity (FDR p-value $=1.56 \mathrm{E}-2)$, and eosinophil migration (FDR p-value $=1.83 \mathrm{E}-2$ ). Nine differentially methylated regions were identified (fwerArea $<0.1$ ) including a region in the promoter of $Z N F 300$ that showed consistent differential DNAm in samples from earlier timepoints in pregnancy and appeared to be driven predominately by effects in the trophoblast cell type. We describe the largest study of fetal sex differences in placenta DNAm performed to date, revealing genes and pathways characterizing sex-specific placenta function and health outcomes later in life.

\section{Introduction}

The placenta is a key organ during pregnancy, performing important functions such as providing nutrients, transferring respiratory gases, and secreting hormones for adequate fetal growth and development ${ }^{1}$. During pregnancy, the placenta grows and changes in composition and function, developing within the mother, but is primarily regulated by the fetal genome ${ }^{1,2}$. Epidemiologic evidence suggests that the periconception and in utero periods are the most vulnerable to environmental factors influencing the susceptibility for several diseases later in life $\mathrm{e}^{3,4}$. The placenta, as the key mediator of the gestational environment, therefore has a significant role in the fetal programming of health outcomes into adulthood ${ }^{5}$. Further, observed differences by sex with respect to prevalence or severity in these health outcomes, as has been observed for autism spectrum disorder (ASD) ${ }^{6}$, schizophrenia ${ }^{7}$, and autoimmune diseases ${ }^{8}$, among many other examples, may be influenced by sex-specific gestational environments that are mediated by sex-specific placenta structure and function.

Some male-female differences during in utero fetal development and childbirth are already known. For example, male fetuses are characterized by higher birth weight, placenta weight, and birth weight to placenta weight ratio 9,10 . Moreover, males have a higher risk of suffering from peri- and postnatal complications ${ }^{11-16}$. Previous studies have demonstrated that male and female fetuses utilize different mechanisms to cope with adverse intrauterine environments ${ }^{17-20}$. For instance, in the presence of chronic maternal asthma growth of female fetuses is reduced, resulting in neonates of smaller size and lower birth weight compared to neonates from healthy pregnancies ${ }^{18,21}$. Any further complication during pregnancy, such as an acute maternal asthma exacerbation, did not decrease their survival rate, indicating a vital adaptation mechanism of female fetuses ${ }^{21,22}$. In contrast, growth of male fetuses appears not to be reduced in the presence of maternal chronic asthma; however, male fetuses experienced a higher rate of adverse outcomes among women who had a severe asthma exacerbation ${ }^{18,20}$.

A growing body of literature has sought to understand the placental molecular mechanisms at play in disease outcomes or complicated pregnancies. Placenta gene expression studies have examined differences in preeclampsia ${ }^{23,24}$, gestational diabetes mellitus ${ }^{25}$, and intrauterine growth restriction (IUGR) ${ }^{26}$. For DNA methylation (DNAm), examples of studies include those examining preterm birth ${ }^{27}$, ASD $^{28}$, and also preeclampsia ${ }^{29}$ and IUGR ${ }^{30}$. There have been some examples in these studies of analyses that explicitly seek to quantify sex-specific mechanisms. For example, gene expression differences, including for various cytokines, have been shown to characterize fetal sex-specific responses to maternal asthma exposure ${ }^{31-33}$. However, these sex-focused analyses are rare, and there have been few studies that have examined sex itself as an "outcome" in analyses, even in normal or non-pathological placentas. Finally, the majority of transcriptomic and DNAm studies of the placenta have not thoroughly accounted for or investigated the role of cell type heterogeneity in phenotypic studies ${ }^{34}$, though a recent study by Yuan et al. ${ }^{35}$ has helped to address this gap by generating DNAm profiles in the four major placenta cell types. Specifically, this study profiled endothelial cells, placental macrophages known as Hofbauer cells, mesenchymal stromal cells and trophoblasts, composed of both syncytiotrophoblasts that interact with maternal blood and cytotrophoblasts that invade the uterine spiral arteries ${ }^{36}$. Overall, the mechanisms by which placental molecular factors act in a sex-specific manner to determine fetal phenotypes, and how these mechanisms act through specific cell types or change with gestational age, remain as critical gaps in our understanding of developmental health. While placental gene expression studies investigating sex-specific differences have been undertaken ${ }^{37-40}$, previous studies of placenta DNAm related to fetal sex ${ }^{41,42}$ have suffered from small sample size.

Here, we present a mega-analysis of publicly available placenta DNAm samples from uncomplicated pregnancies in order to maximize sample size for identification of sex-specific differences. We first sought to characterize sex-specific patterns in DNAm in full term placentas, aiming to identify differentially methylated CpG sites as well as differentially methylated regions (DMRs). We then performed similar analyses using placenta samples from earlier points in pregnancy (1st, 2nd, and 3rd trimesters individually) and evaluated which findings from full term placentas persist across the gestational period. Finally, we examined our results in light of recently published cell type specific placenta DNAm patterns, to understand the degree to which cell type heterogeneity drove our findings and if these patterns could shed light on the mechanism of our identified sites and regions of interest.

\section{Results}

\section{Sex-specific methylation patterns of non-pathological human placenta tissue}

We analyzed sex differences in human placenta DNAm patterns incorporating 783 samples from 14 Illumina 450K array GEO data sets (Fig. 1A,

Supplementary Table 1). Samples from control and/or normal pregnancies were binned into groups according to gestational period. The full term dataset was treated as the discovery dataset, owing to its large sample size. The smaller, less-statistically powered individual trimester datasets were treated as replication cohorts examining the extent to which differentially methylated sites and regions identified at full term persisted throughout the gestational period. In the full term dataset and specific trimester datasets, individual $\mathrm{CpG}$ sites and regions were evaluated for their association with fetal sex, adjusting for dataset-specific 
surrogate variables (see Methods). We verified that the surrogate variables captured batch effects resulting from the many individual studies that contributed to the datasets (Fig. 1B, Supplementary Figs. 1-3).

\section{Differentially methylated $\mathrm{CpG}$ sites by fetal sex in full term placenta samples}

Single site analysis at 445,278 autosomal in 532 full term placenta samples (after QC; see Methods) revealed that a total of 5,212 CpG sites were significantly associated with fetal sex at a genome wide significance threshold of 1E-8 (Fig. 2A, Supplementary Data 1). Of these, cg01382982, harbored in the CpG island promoter of ZNF175, exhibited the largest degree of hypermethylation in males (Fig. 2B; mean difference $=22 \%$, p-value $=9.12 \mathrm{E}-37$ ). In females, the $\mathrm{CpG}$ site achieving genome-wide significance with the highest degree of hypermethylation was cg22905511 (Fig. 2C; mean difference = - $11 \%$, p-value = 2.85E-13), which is located in a CpG island promoter of C5orf63. The majority $(3,793 ; 73 \%)$ of genome-wide significant sites were hypermethylated in male samples; these male-hypermethylated sites tended to exhibit larger effect sizes than those hypermethylated in females (Fig. 2D). Finally, genome-wide significant CpG sites were enriched in GO pathways involved in keratinization, cell differentiation as well as immune cell migration and chemotaxis (Table 1, Supplementary Data 2). There was very minimal overlap between the identified differentially methylated sites and published $450 \mathrm{~K}$-based genome-wide screens of sexassociated DNAm, across a variety of tissue types (Table 2). Though these studies varied in their methodological approach and probe filtering strategies, this result supports the conclusion that the majority of differentially methylated sites identified herein are placenta-specific.

Table 1

Significant (FDR p-value < 0.05) Gene Ontology pathways enriched in differentially

\begin{tabular}{|llll|}
\hline \multicolumn{5}{|c}{ methylated sites from the full term placenta analysis. } \\
\hline GO ID & ONTOLOGY & TERM & FDR p-value \\
\hline GO:0018149 & BP & peptide cross-linking & $7.37 \mathrm{E}-14$ \\
\hline GO:0031424 & BP & keratinization & $7.37 \mathrm{E}-14$ \\
\hline GO:0001533 & CC & ceratinocyte differentiation & $4.07 \mathrm{E}-13$ \\
\hline GO:0070268 & BP & cornification & $1.93 \mathrm{E}-12$ \\
\hline GO:0009913 & BP & epidermal cell differentiation & $7.78 \mathrm{E}-12$ \\
\hline GO:0043588 & BP & skin development & $3.64 \mathrm{E}-08$ \\
\hline GO:0008544 & BP & epidermis development & $1.20 \mathrm{E}-07$ \\
\hline GO:0045095 & CC & keratin filament & $1.67 \mathrm{E}-06$ \\
\hline GO:0030855 & BP & epithelial cell differentiation & $2.41 \mathrm{E}-04$ \\
\hline GO:0060429 & BP & epithelium development & $8.68 \mathrm{E}-04$ \\
\hline GO:0099512 & CC & supramolecular fiber & $3.50 \mathrm{E}-03$ \\
\hline GO:0099081 & CC & supramolecular polymer & $4.55 \mathrm{E}-03$ \\
\hline GO:0099080 & CC & supramolecular complex & $4.55 \mathrm{E}-03$ \\
\hline GO:0048020 & MF & cCR chemokine receptor binding & $1.12 \mathrm{E}-02$ \\
\hline GO:0048245 & BP & eosinophil chemotaxis & $1.39 \mathrm{E}-02$ \\
\hline GO:0008009 & MF & chemokine activity & $1.56 \mathrm{E}-02$ \\
\hline GO:0072677 & BP & eosinophil migration & $1.83 \mathrm{E}-02$ \\
\hline GO:0048513 & BP & animal organ development & $3.51 \mathrm{E}-02$ \\
\hline
\end{tabular}


Table 2

Overlap of significant findings from previous studies of differential DNAm by sex with full term placenta results. Results from previous studies restricted to

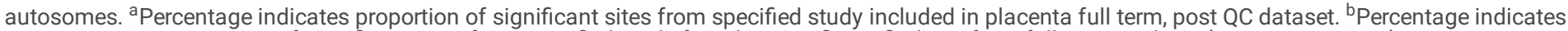
proportion of significant sites from specified study found in significant findings from full term analysis $(p<1 \mathrm{E}-8, n=5,212)$.

\begin{tabular}{|c|c|c|c|c|c|}
\hline Study & Year & Tissue & $\begin{array}{l}\text { \# Significant autosomal CpG sites differential by } \\
\text { sex (Count included in full term, post QC analysis) }\end{array}$ & $\begin{array}{l}\text { \# Significant sites included in post } \\
\text { QC, full term placenta dataset (\%) }\end{array}$ & $\begin{array}{l}\text { Overlap with } \\
\text { placenta full term } \\
\text { results }(\%)^{b}\end{array}$ \\
\hline Price et al. & 2013 & $\begin{array}{l}\text { Peripheral } \\
\text { blood, adult }\end{array}$ & 45 & 40 (88.89\%) & 13 (0.25\%) \\
\hline Hall et al. & 2014 & $\begin{array}{l}\text { Pancreatic } \\
\text { islets }\end{array}$ & 470 & 339 (72.13\%) & $55(1.06 \%)$ \\
\hline Xu et al. & 2014 & $\begin{array}{l}\text { Prefrontal } \\
\text { cortex }\end{array}$ & 13,560 & 13,554 (99.96\%) & 331 (6.35\%) \\
\hline $\begin{array}{l}\text { Inoshita } \\
\text { et al. }\end{array}$ & 2015 & $\begin{array}{l}\text { Peripheral } \\
\text { leukocytes }\end{array}$ & 292 & $292(100 \%)$ & 103 (1.98\%) \\
\hline $\begin{array}{l}\text { Singmann } \\
\text { et al }\end{array}$ & 2015 & $\begin{array}{l}\text { Peripheral } \\
\text { blood }\end{array}$ & 11,010 & 11,008 (99.98\%) & $530(10.2 \%)$ \\
\hline $\begin{array}{l}\text { Spiers et } \\
\text { al. }\end{array}$ & 2015 & Fetal cortex & 521 & $521(100 \%)$ & 103 (1.98\%) \\
\hline $\begin{array}{l}\text { Yousefi et } \\
\text { al. }\end{array}$ & 2015 & Cord blood & 3,031 & $3,031(100 \%)$ & $318(6.10 \%)$ \\
\hline $\begin{array}{l}\text { Martin et } \\
\text { al. }\end{array}$ & 2017 & Placenta & 15 & $1(6.7 \%)$ & $1(0.02 \%)$ \\
\hline \multirow{3}{*}{$\begin{array}{l}\text { Suderman } \\
\text { et al. }\end{array}$} & \multirow[t]{3}{*}{2017} & Cord blood & 11,965 & 11,961 (99.97\%) & $274(5.26 \%)$ \\
\hline & & $\begin{array}{l}\text { Peripheral } \\
\text { blood, } 7 \text { years }\end{array}$ & 13,672 & 13,669 (99.98\%) & 310 (5.95\%) \\
\hline & & $\begin{array}{l}\text { Peripheral } \\
\text { blood, 15-17 } \\
\text { years }\end{array}$ & 12,215 & 12,214 (99.99\%) & 291 (5.58\%) \\
\hline Xia et al. & 2019 & $\begin{array}{l}\text { Prefrontal } \\
\text { cortex, Bulk } \\
\text { Brain }\end{array}$ & 15,417 & $15,048(97.61 \%)$ & 439 (8.42\%) \\
\hline
\end{tabular}

Of the 5,212 differentially methylated sites, 1,285 (24.7\%) were identified as distinguishing full term placenta cell types in a recent paper by Yuan et al. examining cell type specific DNAm patterns in term and early term placentas ${ }^{35}$. This subset of cell type distinguishing probes did not appear to differ markedly from those that did not distinguish cell type in the distribution of effect sizes from the fetal sex comparison (Supplementary Fig. 4). However, GO pathway analysis stratified by probe group type revealed that while keratinization and cell differentiation pathways were shared in the two probe groups, the cell type distinguishing probes were uniquely enriched in chemokine and immune cell migration pathways (Supplementary Data $\mathbf{3}$ and 4).

Persistence of differentially methylated CpG sites across the gestational period

We additionally identified publicly available DNAm samples from preterm placentas, and conducted trimester-specific QC and association analysis using identical methods as used in the full term samples (Supplementary Figs. 1-3; see Methods for classification of placentas into these trimesters). The goal of these analyses of early gestation samples was to understand the extent to which differentially methylated sites identified in full term placenta samples persisted across gestation. Gestational age prediction verified that the full term and trimester-specific datasets $(\mathrm{N}=22,59$, and 37 for Trimesters $1-3$, respectively) captured their intended gestational periods (Fig. 3A). Intuitively, greater correlation was observed in effect sizes as trimesters approached full term; for example the correlation between first trimester and full term effect sizes was $34 \%$ while the correlation between third trimester and full term effect sizes was $80 \%$ (Fig. 3B). Of the 5,209 genome-wide significant CpG sites from the full term analysis (3 CpG sites did not pass QC in $\geq 1$ trimester-specifiC dataset), $194 \mathrm{CpG}$ sites replicated their association with fetal sex in each trimester (Fig. 3C; replication defined as p-value $\leq 0.05$ and consistent direction of effect as seen in full term samples). An example of one of these cross-gestation persistent CpG sites is cg17612569, which maintains a hypermethylated state in male placenta samples of at least $13 \%$ from the first trimester through full term (Table 3, Fig. 3D). A protein-protein interaction network derived from the 194 persistent sites revealed a greater cluster (red) mainly associated to neurogenesis, axonogenesis, gliogenesis and developmental processes and two smaller clusters (green, light green) mainly associated to keratinization and peptide cross-linking. All other networks included less than four gene symbols (Fig. 3E). Full genome-wide association statistics for the analyses from each trimester have been made available (Supplementary Data 5-7; Supplementary Fig. 5). 
Table 3

Top $10 \mathrm{CpG}$ sites from full term analysis (ranked by absolute value of effect size in full term analysis) displaying persistence across the gestational period. Mean Difference (MD) values calculated as mean in male samples - mean in female samples.

\begin{tabular}{|c|c|c|c|c|c|c|c|c|}
\hline CpG Site & $\begin{array}{l}\text { MD, Full } \\
\text { Term }\end{array}$ & $\begin{array}{l}\text { P-value, Full } \\
\text { Term }\end{array}$ & $\begin{array}{l}\text { MD, 3rd } \\
\text { Trimester }\end{array}$ & $\begin{array}{l}\text { P-value, 3rd } \\
\text { Trimester }\end{array}$ & $\begin{array}{l}\text { MD, 2nd } \\
\text { Trimester }\end{array}$ & $\begin{array}{l}\text { P-value, 2nd } \\
\text { Trimester }\end{array}$ & $\begin{array}{l}\text { MD, 1st } \\
\text { Trimester }\end{array}$ & $\begin{array}{l}\text { P-value, 1st } \\
\text { Trimester }\end{array}$ \\
\hline $\operatorname{cg} 02375258$ & 20.4 & $1.46 \mathrm{E}-31$ & 9.1 & 0.0387 & 14 & 0.00378 & 24.1 & 0.000743 \\
\hline cg18237551 & 20 & $8.15 E-35$ & 19.8 & 0.000453 & 22.4 & $1.02 \mathrm{E}-05$ & 18.4 & 0.00035 \\
\hline cg08580836 & 19.9 & $9.05 E-39$ & 18.6 & 0.000253 & 26.3 & $2.83 \mathrm{E}-06$ & 28.2 & 2.03E-05 \\
\hline cg21228005 & 19.6 & $3.4 \mathrm{E}-39$ & 19.6 & 0.00185 & 24.5 & $1.85 \mathrm{E}-06$ & 27.6 & 2.1E-06 \\
\hline cg02343823 & 18.2 & $3.58 \mathrm{E}-36$ & 14.4 & 0.000688 & 18.6 & $1.2 \mathrm{E}-05$ & 17.5 & $3.02 \mathrm{E}-05$ \\
\hline $\operatorname{cg} 04675542$ & 16.5 & $6.38 \mathrm{E}-30$ & 13.3 & 0.00038 & 20.4 & $1.11 \mathrm{E}-05$ & 16.4 & $5.69 \mathrm{E}-05$ \\
\hline cg11291313 & 13.4 & $1.01 \mathrm{E}-33$ & 12 & 0.00164 & 18.2 & $5.29 \mathrm{E}-05$ & 20.4 & $6.01 \mathrm{E}-05$ \\
\hline cg19014419 & 13 & $2.88 \mathrm{E}-34$ & 13.4 & 0.000429 & 17.8 & 4.48E-05 & 18.6 & 0.000183 \\
\hline cg17612569 & 12.6 & 5.93E-119 & 20 & $1.5 \mathrm{E}-13$ & 20.7 & $3.5 \mathrm{E}-20$ & 13.7 & $1.72 \mathrm{E}-07$ \\
\hline cg02325951 & 12.1 & $5.81 \mathrm{E}-182$ & 13.4 & $3.14 \mathrm{E}-16$ & 11.6 & $1.5 \mathrm{E}-12$ & 11.8 & 1.17E-05 \\
\hline
\end{tabular}

\section{Region-based differential DNAm analyses and persistence across gestation}

We also sought to identify DMRs in full term samples and examine the extent to which they persisted across gestation. Nine DMRs were identified as significant beyond a discovery-based threshold (fwerArea value) of 0.1 (Supplementary Fig. 6, Supplementary Data 8). The top-ranked DMR was located in a CpG island promoter of ZNF175 (fwerArea = 0); males exhibited 17\% higher average DNAm relative to females in this region (Fig. 4A). Trimester-specific region-based analyses (Supplementary Data 9-11) were not statistically powered for genome-wide detection. However, these analyses did reveal that the identified ZNF175 promoter region exhibited a similar degree of hypermethylation in males across the gestational period (Fig. 4B-D). In fact, all of the nine full term DMRs exhibited evidence for persistence across gestation via consistent direction of effect across all trimesters (Table 4, Supplementary Figs. 7-14). However, in most cases there was a significantly decreased magnitude of effect size in the individual trimesters. The most robust evidence for persistence, beyond the ZNF175 DMR, was observed for male hypermethylation at ZNF300 promoter (Supplementary Fig. 7) and for female hypermethylation at the C5orf63 promoter (Supplementary Fig. 8). In the latter case, the DMR did not emerge until the 2nd trimester, but persisted thereafter at a similar magnitude.

Table 4

Persistence of genome-wide significant differentially methylated regions from full term analysis across the gestational period. Statistics generated from bumphunter ${ }^{62}$ function in minfi package ${ }^{55}$. Mean Difference (MD) values calculated as mean in male samples - mean in female samples. Final column indic if DMR contained cell type distinguishing probe as identified by Yuan et al. in full term samples.

\begin{tabular}{|c|c|c|c|c|c|c|c|c|c|c|c|c|}
\hline chr & start & end & p.value & fwer & $\begin{array}{l}\text { p.value } \\
\text { Area }\end{array}$ & $\begin{array}{l}\text { fwer } \\
\text { Area }\end{array}$ & $\begin{array}{l}\text { MD, } \\
\text { Full } \\
\text { Term }\end{array}$ & $\begin{array}{l}\text { Nearest } \\
\text { Gene } \\
\text { (location) }\end{array}$ & $\begin{array}{l}\text { MD, } \\
\text { Trimester1 }\end{array}$ & $\begin{array}{l}\text { MD, } \\
\text { Trimester } \\
2\end{array}$ & $\begin{array}{l}\text { MD, } \\
\text { Trimester } \\
\mathbf{3}\end{array}$ & $\begin{array}{l}\text { Contains ce } \\
\text { type } \\
\text { discriminat } \\
\text { probes? }\end{array}$ \\
\hline chr19 & 52074293 & 52074501 & 0 & 0 & 0 & 0 & 0.17 & $\begin{array}{l}\text { ZNF175 } \\
\text { (promoter) }\end{array}$ & 0.24 & 0.0831 & 0.11 & No \\
\hline chr5 & 150284416 & 150284796 & 0 & 0 & 0 & 0 & 0.172 & $\begin{array}{l}\text { ZNF300 } \\
\text { (promoter) }\end{array}$ & 0.21 & 0.212 & 0.159 & Yes \\
\hline chr5 & 126408756 & 126409553 & 0 & 0 & 0 & 0 & -0.0737 & $\begin{array}{l}\text { C5orf63 } \\
\text { (promoter) }\end{array}$ & -0.00439 & -0.101 & -0.101 & Yes \\
\hline chr11 & 2906285 & 2907127 & 0 & 0 & $\begin{array}{l}1.83 \mathrm{E}- \\
06\end{array}$ & 0.007 & 0.0243 & $\begin{array}{l}\text { CDKN1C } \\
\text { (gene body) }\end{array}$ & 0.0325 & 0.0159 & 0.0295 & No \\
\hline chr7 & 86974674 & 86975244 & $\begin{array}{l}2.61 \mathrm{E}- \\
07\end{array}$ & 0.001 & $\begin{array}{l}3.66 \mathrm{E}- \\
06\end{array}$ & 0.014 & -0.0522 & $\begin{array}{l}\text { CROT } \\
\text { (promoter) }\end{array}$ & -0.0514 & -0.0743 & -0.0761 & Yes \\
\hline chr7 & 27169674 & 27171051 & $\begin{array}{l}5.23 \mathrm{E}- \\
07\end{array}$ & 0.002 & $\begin{array}{l}1.05 \mathrm{E}- \\
05\end{array}$ & 0.039 & -0.0372 & $\begin{array}{l}\text { HOXA4 } \\
\text { (promoter) }\end{array}$ & -0.0106 & -0.0035 & 0.00492 & Yes \\
\hline chr4 & 90758120 & 90759203 & $\begin{array}{l}1.05 \mathrm{E}- \\
06\end{array}$ & 0.004 & $\begin{array}{l}1.73 \mathrm{E}- \\
05\end{array}$ & 0.065 & -0.0393 & $\begin{array}{l}\text { SNCA } \\
\text { (promoter) }\end{array}$ & -0.0216 & -0.0138 & -0.00186 & No \\
\hline chr6 & 28641622 & 28642394 & $\begin{array}{l}3.4 \mathrm{E}- \\
06\end{array}$ & 0.013 & $\begin{array}{l}1.83 \mathrm{E}- \\
05\end{array}$ & 0.069 & 0.0329 & $\begin{array}{l}\text { ZBED9 } \\
(58 \mathrm{~Kb} \\
\text { downstream }\end{array}$ & 0.0419 & 0.0692 & 0.0452 & Yes \\
\hline chr9 & 98637332 & 98638444 & $\begin{array}{l}2.61 \mathrm{E}- \\
07\end{array}$ & 0.001 & $\begin{array}{l}2.25 \mathrm{E}- \\
05\end{array}$ & 0.085 & -0.0561 & $\begin{array}{l}\text { ERCC6L2 } \\
\text { (promoter) }\end{array}$ & -0.0427 & -0.0143 & -0.0321 & No \\
\hline
\end{tabular}


Of the nine DMRs, five contained probes that were identified as distinguishing cell type by Yuan et al. (Table 4). Plotting the placenta cell type specific DNAm patterns from this study in the regions defining these DMRs revealed in several instances that particular cell types drive the fetal sex DNAm patterns we observed. For example, male hypermethylation in the ZNF300 promoter is primarily driven by sex differences in trophoblast cell types in this region (Fig. 5A-B). Just as we observed this fetal sex differential DNAm to persist throughout gestation (Fig. 5C, Table 4, Supplementary Fig. 7), the trophoblast signatures remain when examining the 1st trimester samples from Yuan et al. (Fig. 5D). For the remaining four DMRs containing cell type distinguishing probes, cell typerelated inferences are less clear (Supplementary Figs. 15-18), likely owing to the smaller magnitude of DNAm difference in the DMRs. Nonetheless, it can be observed that sex differences in trophoblast DNAm contribute to the DMRs observed at the C5orf63 (Supplementary Fig. 15) and CROT (Supplementary Fig. 16) promoters, with endothelial cells playing a role in the latter case as well, but only in the early gestation time point.

\section{Discussion}

Here we present the largest study to date examining placental DNAm differences by fetal sex. Both single site and region-based analyses revealed large differences according to fetal sex in the placenta methylome. Single site analysis of full term placenta samples demonstrated that a total of $5,212 \mathrm{CpG}$ sites were significantly associated with fetal sex at a genome-wide significance threshold of 1E-8. Pathway analysis results showed that some of the most significantly enriched biological processes were keratinization, cell differentiation, and eosinophil chemotaxis. Replication studies using samples at earlier points in gestation demonstrated evidence that 194 of these $\mathrm{CpG}$ sites are differentially methylated by sex throughout pregnancy. Nine DMRs met a discoverybased threshold of significance, and the top-ranked DMRs at the promoters of ZNF175 and ZNF300, respectively, displayed robust evidence for persistence across the gestational period. In the latter case, cell type specific placenta DNAm data revealed that trophoblast cell types drive the fetal sex association observed in the region.

Our findings of many differentially methylated sites and regions according to fetal sex will contribute to the understanding of the molecular mechanisms driving sex-specific placenta structure and functions. For example, several immune-related pathways were enriched in differentially methylated CpG sites from the full term analysis, which is consistent with several previous investigations of sex-related gene expression differences in placenta ${ }^{37,39,40}$. A recent dataset described by Yuan et $\mathrm{al}^{35}$, which profiled DNAm in sorted placenta cell types, allowed for further characterization of these immune pathway findings. While the Yuan et al. study found no significant differences in estimated cell type proportions between male and female placentas, it is still possible that individual probes exhibit both sex-specific and cell type specific DNAm patterns. Indeed, we found that a significant proportion $(1,285,24.7 \%)$ of our 5,212 fetal sex differentially methylated sites in full term placentas were defined by Yuan et al. to distinguish placenta cell types at full term as well. Stratified pathway analyses considering probes in this group of 5,212 that did and did not distinguish placenta cell types separately revealed that the enrichment of chemokine and immune cell migration pathways seen in the overall group of probes was observed uniquely in the cell-type-distinguishing subset. This result argues that sex-specific immune responses in the placenta are mediated through cell type proportion changes.

Keratinization and cell differentiation pathways were also strongly enriched in the differentially methylated sites at full term, and were enriched in both the cell type distinguishing and non-cell-type-distinguishing probe subsets separately. Similar pathways were found in enrichment studies from a previous investigation of gene expression differences by fetal $\operatorname{sex}^{38}$. These pathways are also remarkably consistent with those previously found to be enriched in a gene set consisting of "core" placenta genes that are conserved across evolution and described as "central for making a placental mammal"43, indicating that sex-specific DNAm in the placenta affects genes that are fundamental to the placenta's function.

A unique feature of our analysis was the exploration of placenta samples from earlier time points in pregnancy. Though these datasets were not conducive to genome-wide discovery owing to their small sample sizes, they did provide an ability to infer which CpG sites and regions identified as differentially methylated in full term samples displayed similar patterns of DNAm throughout the gestational period. We identified $194 \mathrm{CpG}$ sites that met p-value thresholds of differential DNAm, with consistent direction of effect, in all four analyzed datasets (1st Trimester through full term). A protein-protein interaction analysis of the genes nearest to these sites implicated numerous central nervous system-related pathways (neurogenesis, axonogenesis, etc), as well as similar keratinization and peptide cross-linking pathways as seen in the full term analysis. As placenta datasets continue to be generated, it will be increasingly possible to better characterize persistently differentially methylated sites by fetal sex, to a greater degree possible than this study. Identification of these specific sites (and the genes and pathways they implicate) will be necessary to gain a greater understanding of the placenta's role in "programming" sexdiscordant phenotypes throughout the life course $\mathrm{s}^{3-5}$

At the region level, the two top-ranked DMRs from the full term analysis showed robust evidence for persistence across the gestational period. The top-ranked DMR, located in the CpG-island promoter of ZNF175, is characterized by a $17 \%$ hypermethylation in male samples relative to female, implying greater $Z N F 175$ gene expression in female samples. ZNF175 regulates the expression of several chemokine receptors, and has been previously demonstrated to be upregulated in pre-eclamptic vs. healthy placentas ${ }^{23,24}$, though these studies examined the maternal portion of the tissue. Future studies should aim to dissect the potential role of ZNF175 promoter DNAm in fetal placenta in pre-eclampsia, considering both the male hypermethylated DMR described in this study, as well as the interesting intersection of fetal sex and gestational age in pre-eclampsia prevalence. Specifically, a recent large meta-analysis examining fetal sex and various birth outcomes found female fetuses to be associated with preterm pre-eclampsia, but male fetuses to be associated with term and postterm preeclampsia ${ }^{44}$.

The second-ranked DMR at the ZNF300 promoter also displayed male hypermethylation to a similar degree (17\%), along with strong evidence for the persistence of this DNAm difference across the gestational period. ZNF300 is a transcriptional repressor with multiple reported targets ${ }^{45}$. It has been linked to immune cell differentiation 46,47 and tumorigenesis ${ }^{48,49}$, furthering the longstanding narrative noting the similarity in placenta cells and cancer cells with respect to their invasive properties ${ }^{50}$. Along these lines, we found through incorporation of the Yuan et al. placenta cell type data ${ }^{35}$ that fetal sex differences in ZNF300 promoter DNAm are driven by differences seen in the trophoblast cell type, which is primarily responsible for placental invasion ${ }^{51}$.

Page 6/16 
In placenta specifically, it has been previously demonstrated that samples collected from twins discordant for intrauterine growth restriction (IUGR) showed significant hypermethylation at the ZNF300 promoter in the IUGR samples ${ }^{30}$. Our companion paper expands this story further through integration of imputed genotyping and placenta morphology measurements with comprehensive DNAm profiling (whole genome bisulfite sequencing, WGBS) ${ }^{52}$. Specifically, this study also demonstrates the existence of hypermethylation in male placentas at the ZNF300 promoter, but further shows that these DNAm signatures mediate cis and trans (X chromosome) effects of genetic variants on placenta area. Collectively, these findings demonstrate robust evidence for a fetal sex DMR in the ZNF300 promoter that is present across pregnancy and driven by trophoblast cell types, and provide a molecular mechanism for the governance of placenta size by fetal genetic variation. Future (in vitro) work should aim to further investigate the directionality of these phenomena in trophoblast cells specifically, to understand if $\mathrm{CpG}$ island promoter hypermethylation leads to reduced ZNF300 expression (as expected canonically) and the relationship between ZNF300 expression and cell proliferation.

There are several limitations of our approach that need to be recognized. First, we strictly leveraged publicly available data, which have limited annotation. In some cases, we were able to perform quality control or sanity checks to address these shortcomings. For example we used SNP probes on the Illumina $450 \mathrm{~K}$ Array to identify and remove duplicate samples that may have been submitted publicly more than once, and we used a gestational age prediction algorithm to confirm that our sample classification was consistent with that guided by available metadata. But in some cases these checks were not possible, such as in selecting samples from normal/typical pregnancies, which was done solely with the provided metadata. Another limitation was the use of datasets that used the 450K array, as newer technologies to query DNAm now exist. Our companion paper ${ }^{52}$ describes an interrogation of sex-related DNAm patterns via WGBS, a comprehensive query of the methylome, but it is currently not feasible to perform these measurements on a large sample size. A better balance of methylome coverage and sample throughput is struck with the next iteration of the 450K Array, the Methylation EPIC array. Finally, our study utilized bulk placenta samples, which are a mixture of placenta cell types. We did perform several post hoc analyses to disentangle the role of different cell types in our findings. However, future work should aim to interrogate fetal sex DNAm differences directly in individual placenta cell populations to better understand, globally, the extent to which different cell types drive sex-related DNAm differences in the placenta.

Overall, future work should seek to better understand the consequence of fetal sex DNAm differences by establishing connections with additional molecular, phenotypic, and morphological endpoints. For example, paired placenta DNAm and gene expression or simply better integration of these two data types can be used to translate DNAm differences into gene expression changes, particularly in genomic regions outside of CpG island promoters and gene bodies where expectations of these relationships are less well known. In addition, placenta DNAm studies of pregnancy outcomes should be fetal sex-stratified or evaluate an interaction with fetal sex, given the significant differences by fetal sex identified in this study. In a similar vein, studies that collect placenta samples should aim to follow-up subjects throughout childhood and into adulthood whenever possible, to better quantify the potential for fetal sex DNAm differences to "program" sex-discordant health outcomes. Finally, as in our companion paper, future placenta studies should aim to collect placental morphology phenotypes in order to relate observed DNAm changes to placenta size, shape, and structure.

In conclusion, this study presents profound differences in DNAm from full term placentas according to fetal sex at the site and region level. Many of these differences, including strong hypermethylated signals in male samples relative to females at the ZNF175 and ZNF300 promoters, show strong evidence for their existence throughout pregnancy. Further characterization of these DNAm signatures will enable improved understanding of sex-specificity in placenta structure and function, gestational environments, and health outcomes across the life course.

\section{Methods}

\section{Study selection}

We searched the Gene Expression Omnibus (GEO) database on July 19th, 2018 for Illumina 450K microarray data sets containing human placental tissue samples by applying the following search terms: "placental methylation" or "placenta methylation" and limiting to platform types of "methylation profiling by array", "methylation profiling by genome tiling array", and "methylation profiling by SNP array". We manually reviewed the resulting 78 studies and removed any that did not use the $450 \mathrm{~K}$ array or did not make the raw .idat files available. We also restricted our study samples to only those labeled as phenotypically normal or controls by the metadata available in GEO. To assign samples within each study to gestational age (GA) categories of full term, first trimester, second trimester, and third trimester, we used the gestational age variable included in the sample metadata in GEO. Specifically, the first trimester group was defined as $\mathrm{GA}<14$ weeks, the second trimester as $14 \leq \mathrm{GA}<28$ weeks, the third trimester as $28 \leq \mathrm{GA}<37$ weeks, and full term as GA>37 weeks. If a GA variable was not available, we consulted the manuscript associated with the GEO dataset. A total of 14 GEO datasets comprising 783 samples were included in the analysis, including 637 full term, 33 1st trimester, 72 2nd trimester, and 41 3rd trimester samples (Supplementary Table 1). We predicted the gestational age of these samples using the predictAge ( function from the planet $\mathrm{R}$ package $^{53}$ to verify that our sample groups captured their intended points during the gestation period $^{54}$

\section{Array pre-processing, quality control, and batch effect correction}

All analyses were performed using R (v4.0.3) and the minfi R package ( 1.36$)^{55}$ unless otherwise stated. We theorized that some of the placenta samples in the public domain could be duplicates. Hence, from the samples that passed the inclusion criteria, duplicate pairs of samples were identified using the 65 single nucleotide polymorphisms (SNP) probes measured on the 450K array. The sample from the identified pair with the greater number of probes defined as detection $p$-value failures ( $p>0.01$ ) was removed. The preprocessNoob) function ${ }^{56,57}$ was used for background correction and dye-bias equalization. Samples that had low overall intensity (median unmethylated or methylated signal $<11$ ) or had a detection $p$-value $>0.01$ in more than $1 \%$ of probes or probes that had a detection p-value $>0.01$ in more than $10 \%$ of samples were removed. Samples were removed if the reported sex did not match predicted sex generated by the getSex ( function in minfi. We removed probes mapping to sex chromosomes, and also removed autosomal-targeting probes that ambiguously map to sex chromosome locations according to Chen et al. ${ }^{58}$. After these steps the dataset for the full term samples consisted of 445,278 
probes and 532 samples. Finally, quantile normalization was performed, and surrogate variables were estimated using the sva R package ${ }^{59}$ on the resulting $\mathrm{M}$ values. SVs have been shown to capture differences related to batch effects and cell type proportions across samples in a wide variety of simulated settings ${ }^{60}$, and to remove the effects of unwanted sources of technical and biological variation ${ }^{59}$.

We repeated these QC and batch effect correction steps in each trimester dataset individually. For the 1 st trimester dataset, these steps resulted in 444,948 probes and 22 samples available for downstream analysis. For the 2nd trimester, it was 445,331 probes and 59 samples and for the 3rd trimester it was 445,220 probes and 37 samples.

\section{Identification of differentially methylated CpG sites and regions}

To identify differentially methylated individual CpG sites using the limma R package ${ }^{61}$, we modeled M-values as function of fetal sex and the estimated SVs and defined genome-wide significant probes as those with p-values $<1 \mathrm{E}-8$. The bumphunter 0 function ${ }^{62}$ was used to identify differentially methylated regions (DMRs) using the same model. We repeated these same association testing procedures in each trimester individually. Because these datasets were not powered for genome-wide discovery, we did not impose strict statistical significance cutoffs for these analyses. Instead, we looked for evidence of replication of differentially DNAm identified in the full term samples, to quantify the extent to which these differences persisted across the gestational period. We considered a early term probe to replicate the full term result if the fetal sex analysis showed a p-value $<0.05$ and a consistent direction of effect with that observed in the full term samples.

\section{Gene enrichment and pathway analysis}

After identifying differentially methylated $\mathrm{CpG}$ sites and DMRs, we sought to characterize biological pathways enriched in our top findings. All probes reaching a significance threshold of 1E-8 were evaluated for enrichment in Gene Ontology (GO) pathways using the gometh() and topGSA() functions in the R package missMethyl. STRING protein-protein interaction network analyses were also performed after mapping significant $\mathrm{CpG}$ sites to their nearest genes ${ }^{63}$.

\section{Comparison of results to previous DNAm studies of sex}

Significant CpG sites discovered via our analysis were compared to significant CpG sites found in previous human sex-related DNAm studies to determine how many sites had already been identified in the literature. These previous studies analyzed placenta ${ }^{41,64}$ as well as cord blood ${ }^{65,66}$, peripheral blood ${ }^{66-69}$, saliva $^{70}$, prefrontal cortex ${ }^{71,72}$, pancreas ${ }^{73}$, and fetal cortex ${ }^{74}$.

\section{Cell type heterogeneity analyses}

To understand the extent to which our fetal sex differentially methylated sites and regions were driven by cell type heterogeneity, we compared our results to those from a recent study by Yuan et al. examining placenta cell type-specific DNAm patterns ${ }^{35}$. Specifically, we quantified the degree of overlap in differentially methylated sites from our full term analysis with probes identified in the Yuan et al. study as distinguishing cell type at full term, and conducted GO pathway analyses in groups of probes that did and did not overlap with this list separately. We also determined which of the identified DMRs from the full term analysis contained probes from this list, downloaded the data from the Yuan et al. study (GEO ID: GSE159526) and plotted the cell type specific DNAm profiles in these same regions to determine which cell type(s) were driving the DMR result.

\section{Declarations}

\section{Competing Interests}

All authors declare that they have no conflict of interest

\section{Author's Contributions}

SVA conceived the study, performed the data analysis, and wrote the paper. IJY performed the data analysis and wrote the paper. KF performed the data analysis and wrote the paper. TO wrote the paper. MS conceived the study and wrote the paper. All authors contributed to interpretation of results and edited and reviewed the manuscript.

\section{Data Availability}

All placenta datasets used for association testing in this study are available in GEO and listed in Supplementary Table 1. Cell type specific placenta DNAm data described in Yuan et al. ${ }^{35}$ were also downloaded from GEO (GSE159526).

\section{Code Availability}

Scripts for analyses conducted in this study are available at https://github.com/sandrews5/PlacentaDNAm_FetalSex

\section{References}

1. Burton, G. J. \& Fowden, A. L. The placenta: a multifaceted, transient organ. Philos. Trans. R. Soc. B Biol. Sci. 370, 20140066 (2015).

2. Bianco-Miotto, T. et al. Recent progress towards understanding the role of DNA methylation in human placental development. Reprod. Camb. Engl. 152, R23-30 (2016)

3. Barker, D. J. P. The developmental origins of well-being. Philos. Trans. R. Soc. Lond. B. Biol. Sci. 359, 1359-1366 (2004).

4. Gillman, M. W. Developmental origins of health and disease. N. Engl. J. Med. 353, 1848-1850 (2005). 
5. Novakovic, B. \& Saffery, R. The ever growing complexity of placental epigenetics - role in adverse pregnancy outcomes and fetal programming. Placenta 33, 959-970 (2012).

6. Werling, D. M. \& Geschwind, D. H. Sex differences in autism spectrum disorders. Curr. Opin. Neurol. 26, 146-153 (2013).

7. Aleman, A., Kahn, R. S. \& Selten, J.-P. Sex differences in the risk of schizophrenia: evidence from meta-analysis. Arch. Gen. Psychiatry 60, 565-571 (2003).

8. Ngo, S. T., Steyn, F. J. \& McCombe, P. A. Gender differences in autoimmune disease. Front. Neuroendocrinol. 35, 347-369 (2014).

9. Cogswell, M. E. \& Yip, R. The influence of fetal and maternal factors on the distribution of birthweight. Semin. Perinatol. 19, 222-240 (1995).

10. Wallace, J. M., Bhattacharya, S. \& Horgan, G. W. Gestational age, gender and parity specific centile charts for placental weight for singleton deliveries in Aberdeen, UK. Placenta 34, 269-274 (2013).

11. Bracero, L. A., Cassidy, S. \& Byrne, D. W. Effect of gender on perinatal outcome in pregnancies complicated by diabetes. Gynecol. Obstet. Invest. 41, 10-14 (1996).

12. Bekedam, D. J., Engelsbel, S., Mol, B. W. j, Buitendijk, S. E. \& van der Pal-de Bruin, K. M. Male predominance in fetal distress during labor. Am. J. Obstet. Gynecol. 187, 1605-1607 (2002).

13. Zeitlin, J., Ancel, P.-Y., Larroque, B., Kaminski, M., \& EPIPAGE Study. Fetal sex and indicated very preterm birth: results of the EPIPAGE study. Am. J. Obstet. Gynecol. 190, 1322-1325 (2004).

14. Cui, W. et al. Sex differences in birth defects: a study of opposite-sex twins. Birt. Defects Res. A. Clin. Mol. Teratol. 73, 876-880 (2005).

15. Peelen, M. J. C. S. et al. Impact of fetal gender on the risk of preterm birth, a national cohort study. Acta Obstet. Gynecol. Scand. 95, 1034-1041 (2016).

16. Challis, J., Newnham, J., Petraglia, F., Yeganegi, M. \& Bocking, A. Fetal sex and preterm birth. Placenta 34, 95-99 (2013).

17. Vatten, L. J. \& Skjaerven, R. Offspring sex and pregnancy outcome by length of gestation. Early Hum. Dev. 76, 47-54 (2004).

18. Murphy, V. E. et al. Maternal Asthma Is Associated with Reduced Female Fetal Growth. Am. J. Respir. Crit. Care Med. 168, 1317-1323 (2003).

19. Stark, M. J., Clifton, V. L. \& Wright, I. M. R. Neonates Born to Mothers With Preeclampsia Exhibit Sex-Specific Alterations in Microvascular Function. Pediatr. Res. 65, 291-295 (2009).

20. Murphy, V. E., Gibson, P., Talbot, P. I. \& Clifton, V. L. Severe asthma exacerbations during pregnancy. Obstet. Gynecol. 106, 1046-1054 (2005).

21. Clifton, V. L. Review: Sex and the human placenta: mediating differential strategies of fetal growth and survival. Placenta 31 Suppl, S33-39 (2010).

22. Clark, J. M. et al. Effect of maternal asthma on birthweight and neonatal outcome in a British inner-city population. Paediatr. Perinat. Epidemiol. 21, 154162 (2007).

23. Trifonova, E. A. et al. Analysis of the Placental Tissue Transcriptome of Normal and Preeclampsia Complicated Pregnancies. Acta Naturae 6, 71-83 (2014).

24. Kaartokallio, T., Cervera, A., Kyllönen, A. \& Laivuori, K. Gene expression profiling of pre-eclamptic placentae by RNA sequencing. Sci. Rep. 5, (2015).

25. Li, J. et al. A MicroRNA Signature in Gestational Diabetes Mellitus Associated with Risk of Macrosomia. Cell. Physiol. Biochem. Int. J. Exp. Cell. Physiol. Biochem. Pharmacol. 37, 243-252 (2015).

26. Majewska, M. et al. Placenta Transcriptome Profiling in Intrauterine Growth Restriction (IUGR). Int. J. Mol. Sci. 20, (2019).

27. Tilley, S. K. et al. Placental CpG methylation of infants born extremely preterm predicts cognitive impairment later in life. PloS One 13, e0193271 (2018).

28. Schroeder, D. I. et al. Placental methylome analysis from a prospective autism study. Mol. Autism 7, 51 (2016).

29. Leavey, K., Wilson, S. L., Bainbridge, S. A., Robinson, W. P. \& Cox, B. J. Epigenetic regulation of placental gene expression in transcriptional subtypes of preeclampsia. Clin. Epigenetics 10, 28 (2018).

30. Roifman, M. et al. Genome-wide placental DNA methylation analysis of severely growth-discordant monochorionic twins reveals novel epigenetic targets for intrauterine growth restriction. Clin. Epigenetics 8, 70 (2016).

31. Scott, N. M. et al. Placental cytokine expression covaries with maternal asthma severity and fetal sex. J. Immunol. Baltim. Md 1950 182, 1411-1420 (2009).

32. Scott, N. M. et al. The presence of maternal asthma during pregnancy suppresses the placental pro-inflammatory response to an immune challenge in vitro. Placenta 32, 454-461 (2011).

33. Osei-Kumah, A., Smith, R., Jurisica, I., Caniggia, I. \& Clifton, V. L. Sex-specific differences in placental global gene expression in pregnancies complicated by asthma. Placenta 32, 570-578 (2011).

34. Jaffe, A. E. \& Irizarry, R. A. Accounting for cellular heterogeneity is critical in epigenome-wide association studies. Genome Biol. 15, R31 (2014).

35. Yuan, V. et al. Cell-specific characterization of the placental methylome. BMC Genomics 22, 6 (2021).

36. Wang, Y. \& Zhao, S. Vascular Biology of the Placenta. (Morgan \& Claypool Life Sciences, 2010).

37. Cvitic, S. et al. The Human Placental Sexome Differs between Trophoblast Epithelium and Villous Vessel Endothelium. PLoS ONE 8, (2013).

38. Buckberry, S., Bianco-Miotto, T., Bent, S. J., Dekker, G. A. \& Roberts, C. T. Integrative transcriptome meta-analysis reveals widespread sex-biased gene expression at the human fetal-maternal interface. Mol. Hum. Reprod. 20, 810-819 (2014).

39. Gonzalez, T. L. et al. Sex differences in the late first trimester human placenta transcriptome. Biol. Sex Differ. 9, 4 (2018).

40. Sood, R., Zehnder, J. L., Druzin, M. L. \& Brown, P. O. Gene expression patterns in human placenta. Proc. Natl. Acad. Sci. U. S. A. 103, 5478-5483 (2006).

41. Martin, E. et al. Sexual epigenetic dimorphism in the human placenta: implications for susceptibility during the prenatal period. Epigenomics $9,267-278$

(2017). 
42. Gong, S. et al. Genome-wide oxidative bisulfite sequencing identifies sex-specific methylation differences in the human placenta. Epigenetics 13, 228-239 (2018).

43. Dunwell, T. L., Paps, J. \& Holland, P. W. H. Novel and divergent genes in the evolution of placental mammals. Proc. R. Soc. B Biol. Sci. 284, (2017).

44. Broere-Brown, Z. A. et al. Fetal sex and maternal pregnancy outcomes: a systematic review and meta-analysis. Biol. Sex Differ. 11, (2020).

45. Qiu, H. et al. Identification of the DNA binding element of the human ZNF300 protein. Cell. Mol. Biol. Lett. 13, $391-403$ (2008).

46. Xu, J.-H. et al. PU.1 can regulate the ZNF300 promoter in APL-derived promyelocytes HL-60. Leuk. Res. 34, 1636-1646 (2010).

47. Cai, J. et al. ZNF300 knockdown inhibits forced megakaryocytic differentiation by phorbol and erythrocytic differentiation by arabinofuranosyl cytidine in K562 cells. PloS One 9, e114768 (2014).

48. Wang, T. et al. Overexpression of the human ZNF300 gene enhances growth and metastasis of cancer cells through activating NF-kB pathway. J. Cell. Mol. Med. 16, 1134-1145 (2012).

49. Yu, S. et al. ZNF300 promotes chemoresistance and aggressive behaviour in non-small-cell lung cancer. Cell Prolif. 53, e12924 (2020).

50. Murray, M. J. \& Lessey, B. A. Embryo implantation and tumor metastasis: common pathways of invasion and angiogenesis. Semin. Reprod. Endocrinol. 17, 275-290 (1999).

51. Knöfler, M. et al. Human placenta and trophoblast development: key molecular mechanisms and model systems. Cell. Mol. Life Sci. CMLS76, 3479-3496 (2019).

52. Ladd-Acosta, C. et al. Placenta DNA methylation at ZNF300 is associated with fetal sex and placental morphology. bioRxiv 2021.03.05.433992 (2021) doi:10.1101/2021.03.05.433992.

53. Yuan, V. et al. Accurate ethnicity prediction from placental DNA methylation data. Epigenetics Chromatin 12, 51 (2019).

54. Lee, Y. et al. Placental epigenetic clocks: estimating gestational age using placental DNA methylation levels. Aging 11, 4238-4253 (2019).

55. Aryee, M. J. et al. Minfi: a flexible and comprehensive Bioconductor package for the analysis of Infinium DNA methylation microarrays. Bioinforma. Oxf. Engl. 30, 1363-1369 (2014).

56. Fortin, J.-P. et al. Functional normalization of 450k methylation array data improves replication in large cancer studies. Genome Biol. 15, 503 (2014).

57. Triche, T. J., Weisenberger, D. J., Van Den Berg, D., Laird, P. W. \& Siegmund, K. D. Low-level processing of Illumina Infinium DNA Methylation BeadArrays. Nucleic Acids Res. 41, e90 (2013).

58. Chen, Y. et al. Discovery of cross-reactive probes and polymorphic CpGs in the Illumina Infinium HumanMethylation450 microarray. Epigenetics 8, 203209 (2013).

59. Leek, J. T., Johnson, W. E., Parker, H. S., Jaffe, A. E. \& Storey, J. D. The sva package for removing batch effects and other unwanted variation in highthroughput experiments. Bioinformatics 28, 882-883 (2012).

60. McGregor, K. et al. An evaluation of methods correcting for cell-type heterogeneity in DNA methylation studies. Genome Biol. 17, 84 (2016).

61. Ritchie, M. E. et al. limma powers differential expression analyses for RNA-sequencing and microarray studies. Nucleic Acids Res. 43, e47-e47 (2015).

62. Jaffe, A. E. et al. Bump hunting to identify differentially methylated regions in epigenetic epidemiology studies. Int. J. Epidemiol. 41, 200-209 (2012).

63. Szklarczyk, D. et al. STRING v11: protein-protein association networks with increased coverage, supporting functional discovery in genome-wide experimental datasets. Nucleic Acids Res. 47, D607-D613 (2019).

64. McCarthy, N. S. et al. Meta-analysis of human methylation data for evidence of sex-specific autosomal patterns. BMC Genomics 15, 981 (2014).

65. Yousefi, P. et al. Sex differences in DNA methylation assessed by $450 \mathrm{~K}$ BeadChip in newborns. BMC Genomics 16, (2015).

66. Suderman, M. et al. Sex-associated autosomal DNA methylation differences are wide-spread and stable throughout childhood. (2017).

67. Price, M. E. et al. Additional annotation enhances potential for biologically-relevant analysis of the Illumina Infinium HumanMethylation450 BeadChip array. Epigenetics Chromatin 6, 4 (2013).

68. Inoshita, M. et al. Sex differences of leukocytes DNA methylation adjusted for estimated cellular proportions. Biol. Sex Differ. 6, (2015).

69. Singmann, P. et al. Characterization of whole-genome autosomal differences of DNA methylation between men and women. Epigenetics Chromatin 8,43 (2015).

70. Liu, J., Morgan, M., Hutchison, K. \& Calhoun, V. D. A study of the influence of sex on genome wide methylation. PloS One 5, e10028 (2010).

71. Xu, H. et al. Sex-biased methylome and transcriptome in human prefrontal cortex. Hum. Mol. Genet. 23, 1260-1270 (2014).

72. Xia, Y. et al. Sex-differential DNA methylation and associated regulation networks in human brain implicated in the sex-biased risks of psychiatric disorders. Mol. Psychiatry (2019) doi:10.1038/s41380-019-0416-2.

73. Hall, E. et al. Sex differences in the genome-wide DNA methylation pattern and impact on gene expression, microRNA levels and insulin secretion in human pancreatic islets. Genome Biol. 15, 522 (2014).

74. Spiers, H. et al. Methylomic trajectories across human fetal brain development. Genome Res. 25, 338-352 (2015).

\section{Figures}



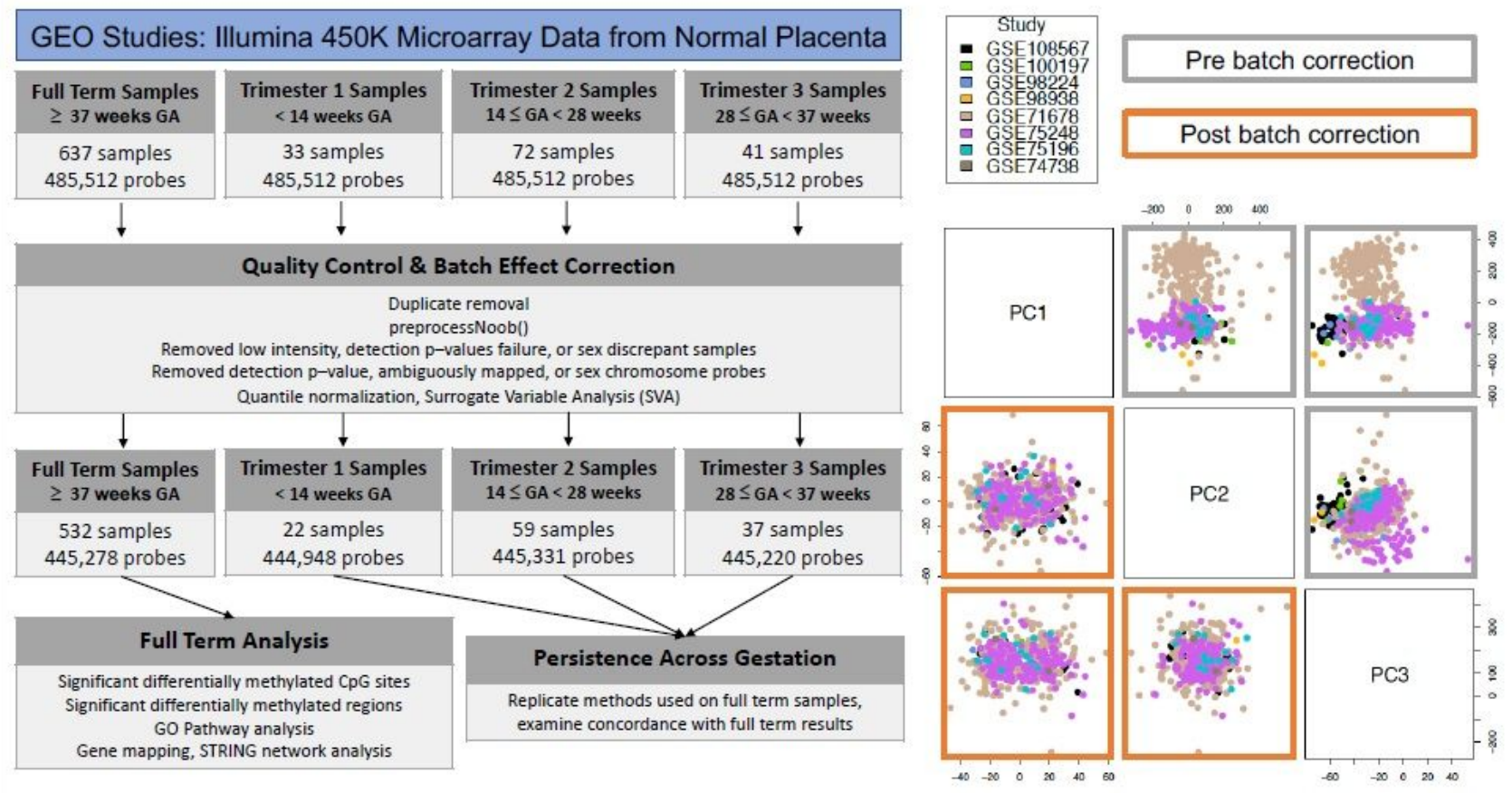

Figure 1

Methods overview and batch effect correction. a Illumina 450K datasets of fetal placenta samples were downloaded from GEO and restricted to those from normal/typical pregnancies. Samples were classified into 4 groups according to annotated gestational age, and each dataset was processed through identical subject and probe level QC steps. Single site and region-based analyses were performed in full term samples first, and then also performed in individual trimester datasets to examine replication of full term findings. See Methods for additional details. b Principal component plot depicting DNAm M-values (top triangle) and residuals from model of M-values regressed onto estimated surrogate variables (bottom triangle) in full term dataset. Points colored by GEO ID. 


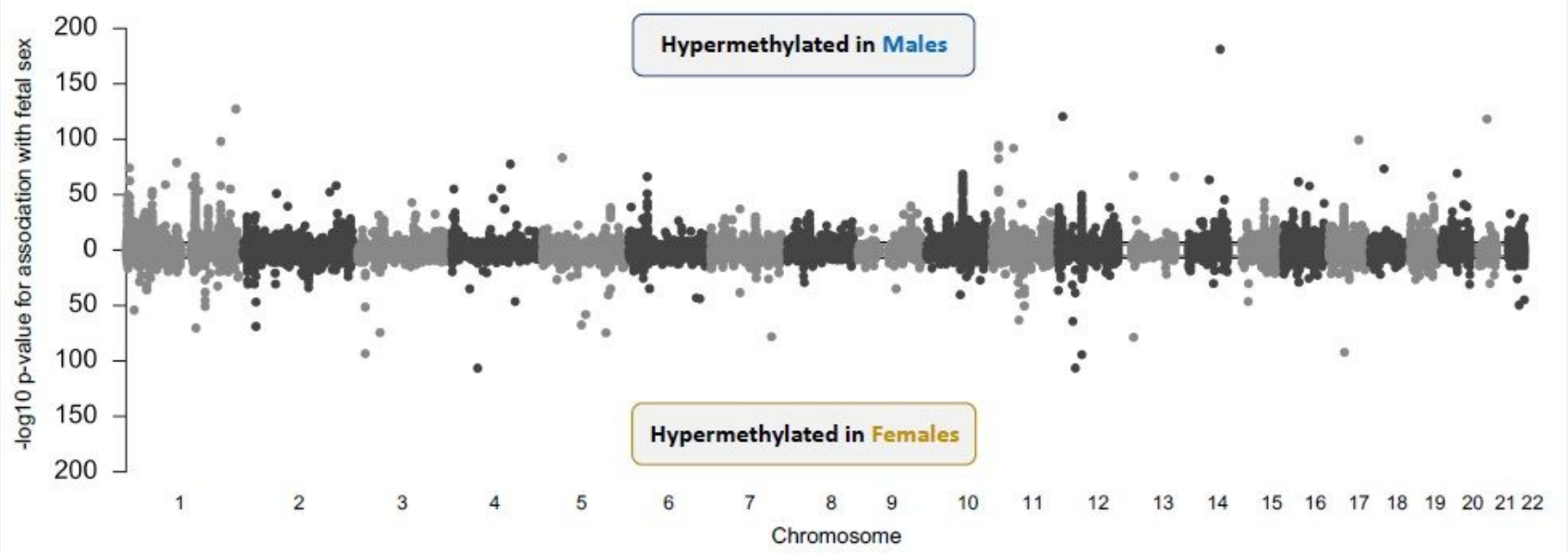

B

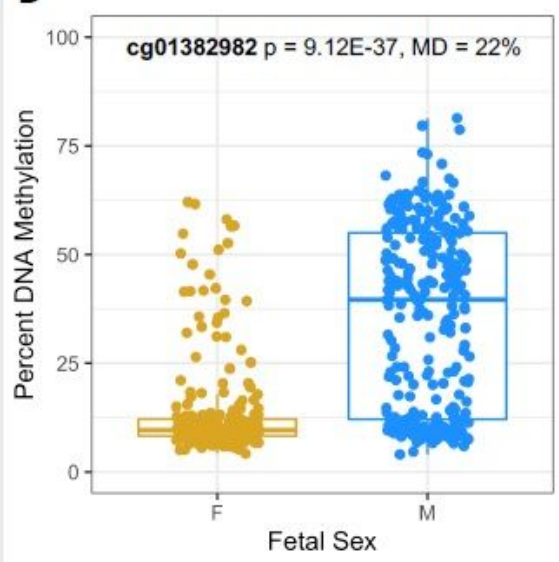

C

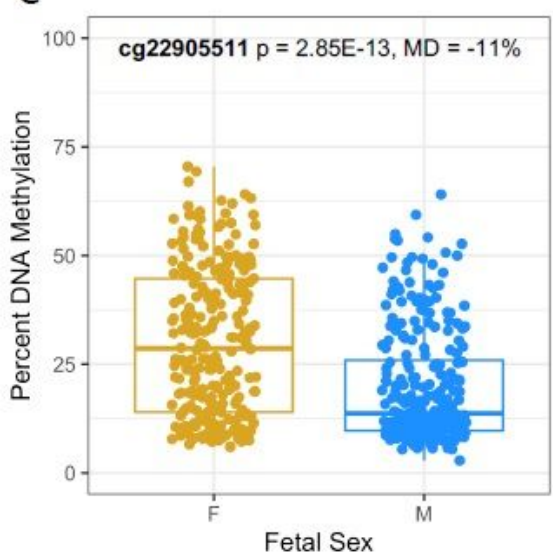

D

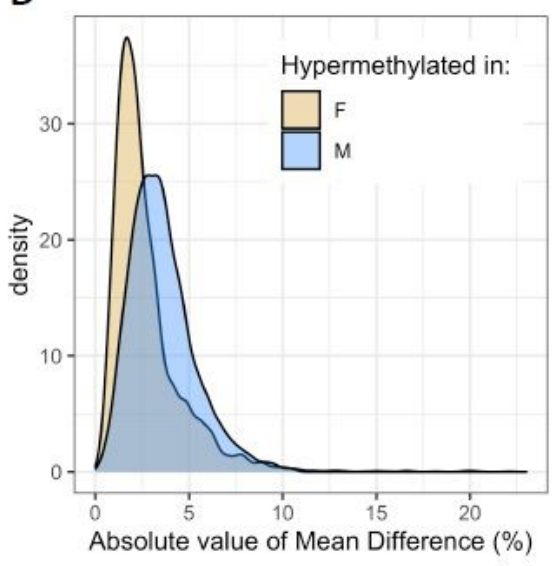

Figure 2

Single site plots. a The manhattan plot provides a global view of the CpG sites from the full term samples. The x-axis represents genomic position, and the $y$ axis represents the - $\log 10$ of the association's p-value. Each point is a $\mathrm{CpG}$ site. b Boxplots of percent methylation in males (blue) and females (gold) at cg01382982, the genome-wide significant CpG site at which males were most hypermethylated relative to females (Mean Difference, MD = 22\%). c Boxplots of percent methylation in males (blue) and females (gold) at cg22905511, the genome-wide significant CpG site at which females were most hypermethylated relative to males (Mean Difference, MD $=-11 \%$ ). $d$ Distribution of absolute value of mean differences in sites hypermethylated in males (blue, $\mathrm{n}=3,793$ ) and sites hypermethylated in females (gold, $n=1,419)$. 


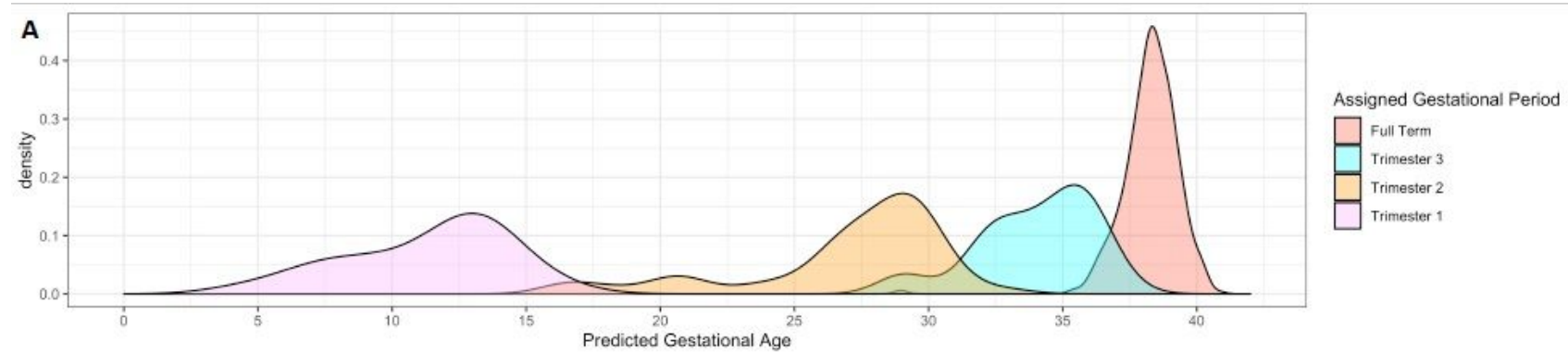

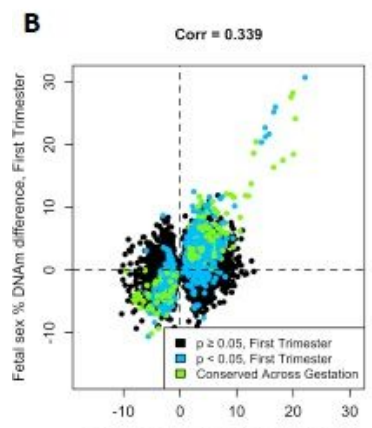

D

Fetal sex \% DNAm difference, Full Term

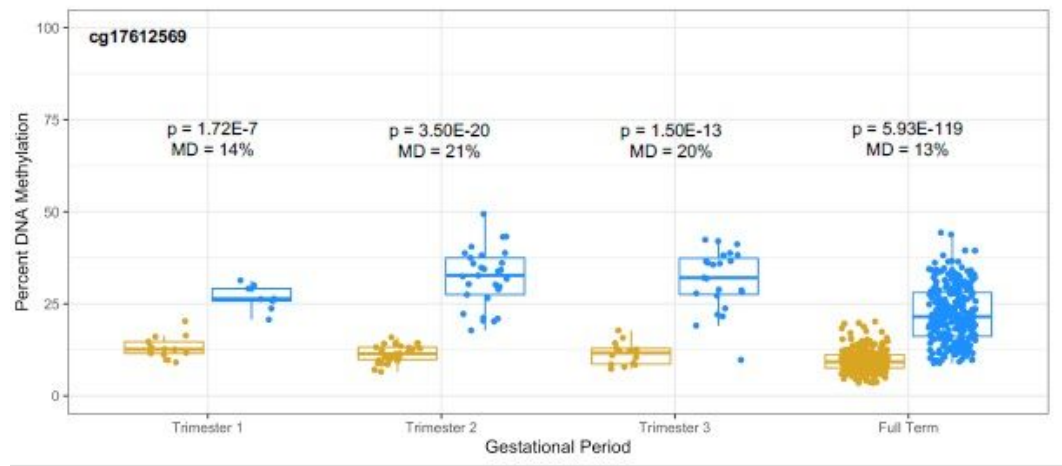

C
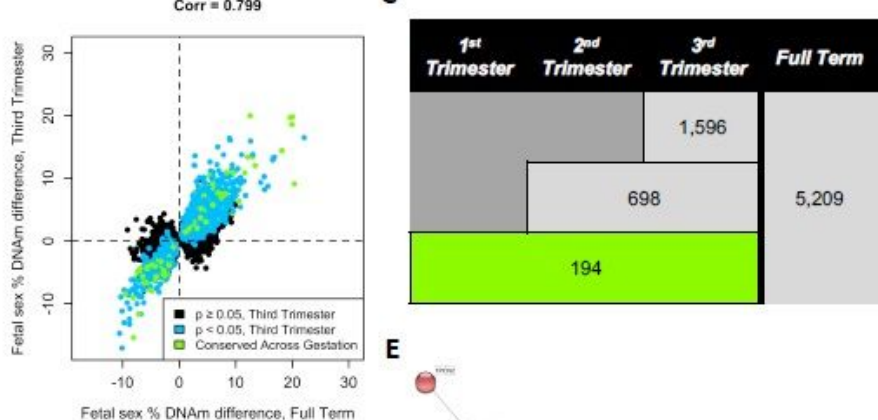

e

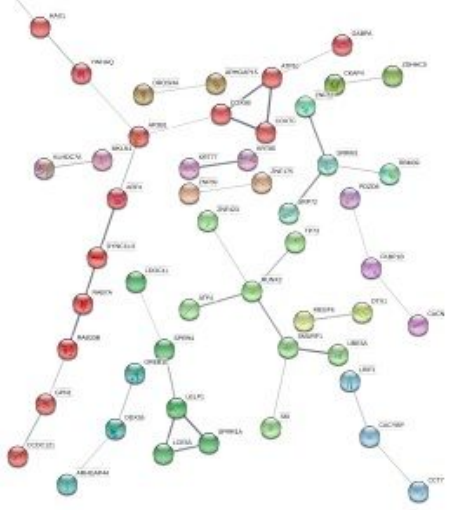

Figure 3

Persistence across the gestational period. a Distribution of predicted gestational age (via predictAge( ) function from the planet $\mathrm{R}$ package) in full term and individual trimester datasets $\mathrm{b}$ The persistence correlation analysis shows plots of DNA methylation differences for the three trimesters. The green colored points are the $\mathrm{CpG}$ sites that were conserved across gestation. The blue colored points represent the CpG sites that were considered significant for each trimester. The correlation values between the trimester and the full term analysis increase as the full term is approached. c Persistence of hits discovered from the full term placentas throughout gestation. If the significant site from the full term had a p value less than 0.05 and had the same direction of association in the preterm analysis, then it was considered "persistent." $d$ Comparison of female and male fetuses' methylation values at different points during gestation. cg17612569 was consistently significant at all time points. e STRING network analysis based on genes mapped from persistent single sites shows proteinprotein interactions. Each node is a protein. The higher confidence scores for the interactions are represented by the darker lines. 

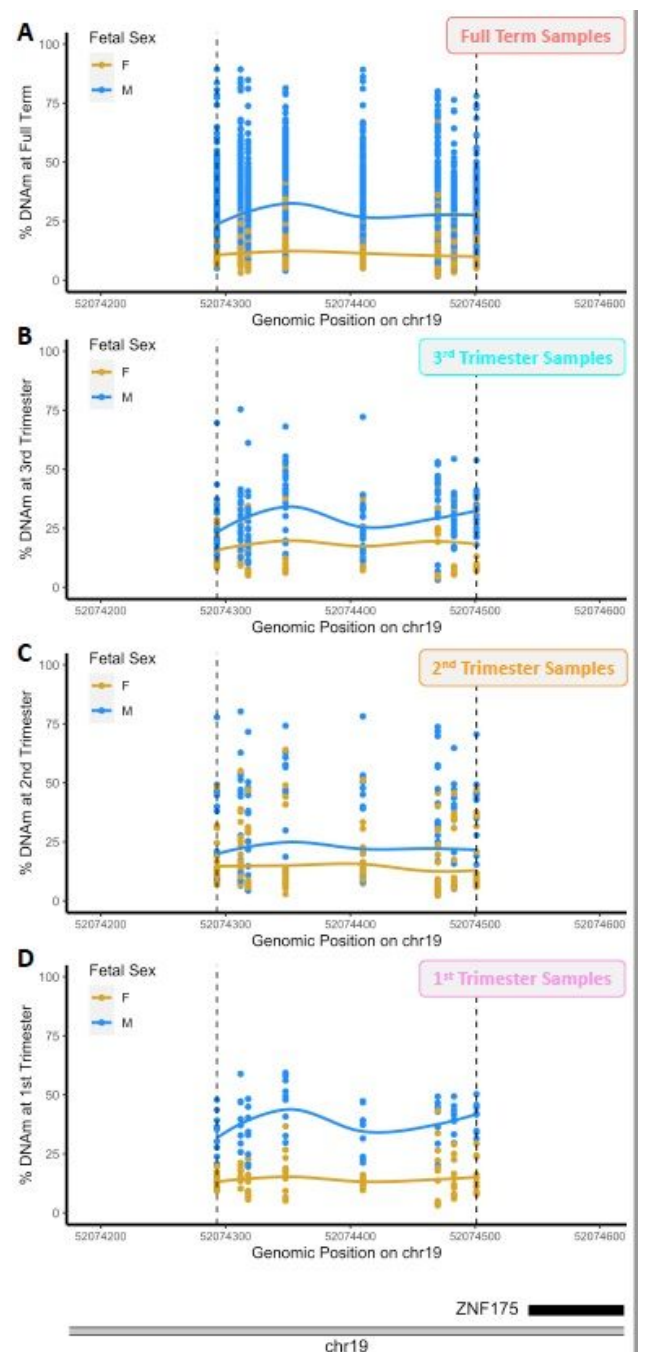

\section{Figure 4}

DMR at the ZNF175 promoter across the gestational period. Top-ranked DMR from full term analysis at ZNF175 promoter. DMRs are plotted as percent methylation as a function of genomic position. Dots indicate samples and solid lines indicate smooth lines through male sample values (blue) and female sample values (gold). Actual identified DMR region indicated via dashed lines. a Full Term Samples. b 3rd Trimester Samples c 2nd Trimester Samples d 1 stTrimester Samples. 


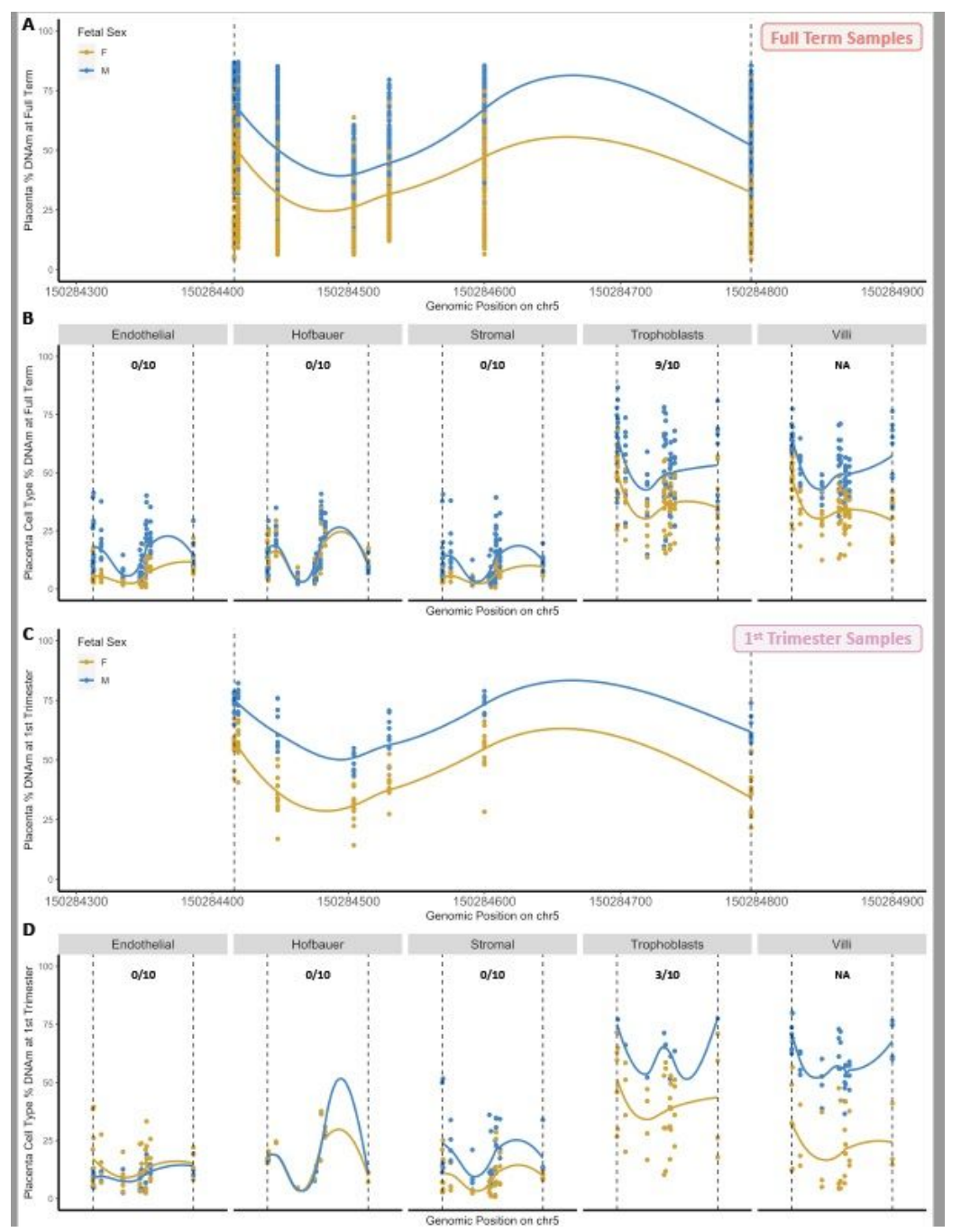

\section{Figure 5}

Cell type heterogeneity at the ZNF300 promoter. DMRs are plotted as percent methylation as a function of genomic position. Dots indicate samples and solid lines indicate smooth lines through male sample values (blue) and female sample values (gold). Actual identified DMR region indicated via dashed lines. a DMR identified at ZNF300 promoter on chromosome 5 in full term analysis, plotted using full term samples from present study. $b$ - $d$ The same region, but plotted using samples from different datasets. b Term samples and individual placenta cell types from Yuan et al. c 1st Trimester samples from present study. $\mathrm{d} 1$ st trimester samples and individual placenta cell types from Yuan et al. In b + d, fractions indicate the proportion of probes in the region that were annotated by Yuan et al. as distinguishing that cell type in full term and 1st trimester samples, respectively. Villi samples were not evaluated in this manner by Yuan et al. as this is the unsorted bulk tissue.

\section{Supplementary Files}

This is a list of supplementary files associated with this preprint. Click to download.

- Supplementarylnformation.pdf

- SupplementaryData1.csv.zip

- SupplementaryData2.csv

- SupplementaryData3.csv

- SupplementaryData4.csv

- SupplementaryData5.csv.zip

- SupplementaryData6.csv.zip

- SupplementaryData7.csv.zip

- SupplementaryData8.csv

- SupplementaryData9.csv

- SupplementaryData10.csv 
- SupplementaryData11.csv

Page 16/16 